\title{
Influencia en el consumo del alcohol y tabaco en el adolescente
}

\author{
Irma Ramírez Castro \\ irma.ramirez@umich.mx \\ Delia Lilia Velazco Capistrán \\ Delia.velazco@umich.mx \\ Morelia, Michoacán México.
}

\section{RESUMEN}

En la presente investigación se abordará un tema que ha sido recurrente en la actualidad debido a las diferentes consecuencias que trae consigo: las drogas. Se realizará un recurrido acerca de los tipos de drogas que se consumen en mayor medida, los factores y causas de riesgo que pueden influir en la decisión de ingerir estas sustancias, así como diversas estadísticas que nos alumbren acerca cómo se encuentra hasta este momento la situación en el país con respecto a este problema.

El propósito de este trabajo, a través de un estudio descriptivo, transversal, será realizar una aproximación a las drogas legales que son ingeridas en la Escuela Preparatoria "José María Morelos y Pavón” así como los efectos que estas pueden traer consigo. Donde se concluye que la sustancia licita con mayor frecuencia de consumo es el cigarro, y las consecuencias de ambas (alcohol y tabaco) traen consigo fatiga, resequedad de garganta, así como falta de retención, está última de gran relevancia en el proceso de enseñanza-aprendizaje de los adolescentes.

Palabras Claves: drogas; drogas licitas; adolescentes; tabaco; alcohol. 
Influencia en el consumo del alcohol...

\title{
Influence on the consumption of alcohol and tobacco in adolescents
}

\begin{abstract}
This research will address a theme that has been recurrent until today due to the different consequences it brings: the drugs. We will talk about the types of drugs that are used the most, risk factors that influence to consume drugs, and statistics that speak of the country's situation with regard to drugs.

The purpose of this work, through a descriptive study, it will be closer to legal drugs what are ingested by students at high school "José María Morelos y Pavón" and the consequences that it bring. It is concluded that the most consumed legal drug is the cigarettes. But the effects of alcohol and cigarettes produce fatigue, dry throat and nonreteciòn in learning. This last is very important in the theaching-learning of students.
\end{abstract}

Keywoeds: drugs; legal drugs; students; alcohol; cigarettes. 


\section{INTRODUCCIÓN}

En la actualidad, el consumo de drogas es una problemática que afecta a la sociedad, el cual trae consigo diferentes consecuencias tanto físicas como sociales y, en algunos casos, psicológicas en los individuos. Este ha sido considerado un tema de interés por diversos autores (Villatoro et al., 2016; Uribe, Verdugo y Zacarías, 2011; Pilatti et al., 2019 , etc.) debido al incremento considerable que ha alcanzado en los últimos años el consumo de estas sustancias, principalmente entre los adolescentes, los cuales recurren a la prueba de estas como un proceso de aprendizaje relacionado con la dinámica cultural y la imitación de su entorno (Uribe et al., 2011; Pilatti et al. 2019).

La Organización Mundial de la Salud (OMS) define el concepto de 'droga' desde dos disciplinas: en medicina "se refiere a toda sustancia con potencial para prevenir o curar una enfermedad o aumentar la salud física o mental” (1994, p. 33); y en farmacología "como toda sustancia química que modifica los procesos fisiológicos y bioquímicos de los tejidos o los organismos". (1994, p. 33), es decir, estas definiciones se encuentran encaminadas, principalmente, hacía los medicamentos utilizados para tratar enfermedades de diversas indoles de manera legal, los cuales necesitan receta médica para su distribución; sin embargo, hay individuos que recurren a estas medicinas por los efectos que generan, pero son conseguidos por otros medios, es decir, sin una receta médica que autorice la compra, esto es, de manera ilegal; por consiguiente entendemos que la definición de 'droga' planteada por la OMS nos guía hacia una dirección que nos refiere a medicamentos; no obstante, aunque el consumo de este tipo de drogas esté en aumento, no es el único del que hablaremos, ya que podemos encontrar otros tipos de sustancias, coloquialmente, conocidas como drogas (los ejemplos podrían ser: alcohol, tabaco, marihuana, cocaína, etc.), las cuales son consumidas, cada vez en mayor medida, por la sociedad. Por tal razón, recurriremos a otra definición también dada por la OMS, 'sustancia o droga psicoactiva':

Sustancia que, cuando se ingiere, afecta a los procesos mentales, p. ej., a la cognición o la afectividad. Este término y su equivalente, sustancia psicotrópica, son las expresiones más neutras y descriptivas para referirse a todo el grupo de sustancias, legales e ilegales, de interés para la política en materia de drogas (1994, p. 58). 
Ambos términos son de relevancia, debido al incremento considerable que se ha tenido en el consumo de drogas, tanto médicas como psicoactivas. Sin embargo, aquellas sustancias que sobresalen por causa del alto número de individuos que las ingieren son: el alcohol, el tabaco, la marihuana, y son precisamente los jóvenes los principales en recurrir a ellas (Gómez, Landeros, Noa y Patricio, 2017; Villatoro et al., 2014; Villatoro et al., 2016).

Según Villatoro et al., (2016) en una investigación realizada en México, donde se abarcan diversos estados del país, señalan que la edad promedio en el inicio del consumo de alcohol es de 12.6 años; mientras que, del tabaco, aproximadamente, es de 13 años. Esto nos dice que es en la adolescencia donde comienza este grave problema, siendo esta una etapa vulnerable en el desarrollo de los jóvenes. Los factores que desencadenan esta problemática son diversos: ya sea por influencia familiar, integración social, observar a sus pares, etc. (Sánchez et al., 2014; Pilatti et al., 2019), y son razones por las cuales los adolescentes toman la decisión de ingerir bebidas alcohólicas y cigarros por primera vez en su vida, lo que provoca, generalmente, el uso regular de estas sustancias.

En los resultados de la Encuesta Nacional de Adicciones (ENA) llevada a cabo en 2011, nos señalan que la población de 12 a 65 años alguna vez en su vida ha ingerido bebidas alcohólicas con un 71.3\%; mientras que en la Encuesta Nacional de Consumo de Drogas, Alcohol y Tabaco (ENCODAT) 2016-2017, los resultados son del 71.0\%, lo cual nos indica que el consumo de alcohol se ha mantenido estable en los últimos años, $\mathrm{y}$, a pesar de que se puede observar con optimismo el hecho de que no haya aumentado el porcentaje de consumo, no significa que tengamos que restar importancia al elevado número de individuos que han ingerido bebidas alcohólicas, aunque, claro, el rango de edad es muy amplio.

Por otro lado, en el tabaco hemos encontrado diferencias significativas: en los resultados de la ENA (2011) observamos que el 21.7\% de la población eran fumadores activos (17.3 millones); mientras que, en ENCODAT (2016-2017) la cifra se redujo considerablemente con un 17.6\% (14.9 millones), esto es un gran avance con respecto al consumo de tabaco donde más del $4 \%$ de individuos ha logrado apartar este vicio.

Otro resultado de relevancia es el de las drogas, donde también se encuentran diferencias importantes, pero con respecto al aumento en el consumo de estas: en la 
ENA (2011) encontramos que, en la población total, el consumo de cualquier droga era de $7.8 \%$, las drogas ilegales del $7.2 \%$ y en la marihuana del 6\%; pero en ENCODAT (2016-2017) las diferencias son significativas, el consumo de cualquier droga es de $10.3 \%$, de drogas ilegales un 9.9\%, mientras que la marihuana alcanzó un 8.6\%.

Con lo anterior podemos observar que, con respecto al alcohol, no ha ocurrido una diferencia remarcable entre los años 2011 y 2016, y, a pesar de que el número es bastante elevado, es significativo que durante cinco años no haya aumentado, y sí descendido un 0.3\%; sin embargo, debemos mencionar el cambio notable, tanto en el consumo de tabaco como de las drogas: el primero con una evolución positiva con respecto a la disminución en su consumo, con al menos un 4\% de la población; mientras que el porcentaje correspondiente a drogas aumenta de manera continua, al ingerirse por lo menos un $2 \%$ más, porcentaje en el que, si no se toman medidas, puede ir aumentando gradualmente.

Sin embargo, centrándonos en los adolescentes de entre 12 y 17 años, según ENCODAT (2016-2017) con respecto al alcohol, el consumo de esta sustancia es de un $39.8 \%$; mientras que del cigarro, el $4.9 \%$ de jóvenes fuma tabaco actualmente, es decir, 684 mil adolescentes mexicanos; y, finalmente, con respecto a las drogas, el consumo (alguna vez en que hayan sido ingeridas) aumentó casi el doble desde el 2011 al 2016, de $3.3 \%$ al $6.4 \%$ en cualquier droga, de $2.9 \%$ a $6.2 \%$ en drogas ilegales y de $2.4 \%$ a $5.3 \%$ en marihuana. Ante esto, entendemos que el alcohol sigue siendo la principal sustancia ingerida, sin embargo, el consumo de drogas supera al de el tabaco en la actualidad, donde según el ENCODAT, la marihuana es la principal droga a la que se recurre. Ahora bien, aunque son diversos los factores que han influido en la decisión del adolescente de consumir drogas, un elemento a tomar en cuenta es la percepción de riesgo que se tiene de estas.

Con respecto a las tres principales sustancias, antes mencionadas, tanto el alcohol como el tabaco son consumidos en gran medida por su status de "legalidad", esto es, aquellos que tienen permitidos su comercialización (OMS, 1994; Barreto, Pérez, Roa, López y Rubiano, 2015) por lo tanto, son más fáciles de conseguir, especialmente para reuniones donde las bebidas van, generalmente, acompañadas de cigarros, $\mathrm{y}$, de igual manera, al ser legales estas sustancias son percibidas como un menor riesgo para la salud, lo cual genera un incremento en el uso de estas (Uribe et al., 2011; Méndez-Ruiz, de Jesús 
Alonso-Castillo, Alonso-Castillo, Uribe-Alvarado y Armendáriz-García, 2015); en cambio, la marihuana aún se encuentra en proceso de legalización, sin embargo, su consumo influye, al igual que con el alcohol y el tabaco, con la concepción de peligrosidad de esta droga, entre menor idea de peligro mayor consumo.

Con lo anterior, nos referimos a que la percepción de riesgo del adolescente influye de determinada manera en la decisión de los jóvenes de ingerir cualquier tipo de sustancias. Uribe et al., (2011), señalan que el consumo del alcohol, tabaco y otras drogas es proporcional con la percepción de peligro que se tiene al ingerir cualquiera de estas. Es decir, el consumo de alcohol y de tabaco ha aumentado en los últimos años debido a que la sensación de peligrosidad que generan es cada vez menor debido al uso recurrente de estas sustancias.

Méndez-Ruiz et al., (2015) en su investigación concuerdan con Uribe et al., esto es, ellos también indican que aquellos adolescentes que no han ingerido alcohol tienen una mayor percepción de riesgo con respecto a este, lo cual nos dice que, de cierta manera, al no haber ingerido bebidas alcohólicas se encuentran protegidos por su creencia acerca de lo peligroso que es el alcohol, así como sus efectos.

Mientras que las drogas, si bien ha aumentado su demanda, no alcanzan al alcohol y al tabaco porque su factor de riesgo aún es considerable para los adolescentes (Uribe et al., 2011). Sin embargo, y como ya observamos un par de párrafos antes, el consumo de drogas ha aumentado en gran medida, superando (aunque por poco) al tabaco, lo cual nos dice que entre más se ingieran cualquier tipo de drogas (centrándonos en marihuana, cocaína, inhalantes, etc.), su percepción de riesgo irá disminuyendo.

De igual modo, en el reporte de la encuesta correspondiente a las drogas de ENCODAT, mencionan que la percepción de riesgo del adolescente juega un papel fundamental con respecto al consumo de estas, donde aquellos que no han probado aún ningún tipo de drogas encuentran su consumo bastante arriesgado: el ingerir cocaína se considera muy peligroso con un $71.3 \%$, los inhalables con un $70.7 \%$ y la marihuana con un $39.9 \%$; mientras que al cuestionárseles a aquellos que ya han probado estas sustancias, se encuentra que $62.8 \%$ considera arriesgado el consumo de cocaína, el de inhalables se reduce a un $66.9 \%$, sin embargo, el resultado más drástico ocurre con la marihuana, siendo este de un $18.7 \%$ (2017, p. 78), es decir, al probar la marihuana se genera una reducción notable en la percepción que tiene el adolescente acerca del riesgo que 
provoca esta droga, y lo cual genera, como ya se mencionó, un aumento considerable en su consumo.

Entendemos de esta manera que la percepción de riesgo es un factor de relevancia en la decisión de ingerir cualquier tipo de droga, aunque cabe resaltar que no es el único; anteriormente se han mencionado diversos factores que pueden ser determinantes en los adolescentes para comenzar con esta práctica, entre ellos que los jóvenes recurren a este consumo como parte de su manera de relacionarse con otros (Uribe et al., 2011; Pilatti et al. 2019).

En la investigación de Sánchez, Villareal, Ávila, Vera y Musito (2014) mencionan que hay diferentes componentes que influyen en la voluntad de recurrir a estas sustancias: como lo serían los factores contextuales de familia, escuela y comunidad; así como los factores personales como autoestima, sintomatología depresiva y expectativas académicas. Algunos de estos elementos los clasifican como factores de riesgo y otros de protección: la autoestima social, en esta investigación, fue considerada como factor de riesgo debido al aumento en el consumo de drogas por parte de los adolescentes para poder sentirse parte de un determinado grupo de amigos e integrarse de una manera más sencilla; mientras que el autoestima escolar la relacionan como un factor de protección donde el adolescente al considerarse como un buen alumno, obtener buenas calificaciones y ser estimado por sus maestros, tiende a tener una menor dependencia hacia el consumo de sustancias.

Sin embargo, hay algo que no podemos perder de vista: el contexto familiar es de suma importancia para el desarrollo de los jóvenes, tal y como lo expresan Sánchez et al., "las características del funcionamiento familiar se relacionan, en gran parte, con el consumo de drogas de los hijos/as adolescentes porque influyen en el desarrollo de su autoestima, tanto de protección (autoestima escolar) como la de riesgo (autoestima social)" (Sánchez et al., 2014, p. 75).

En la Investigación de Vallescar, Moreno Sarquella, Subiras, Martín (1986) reflexionan realizando una serie de cuestionamiento ¿Por qué los estudiantes fuman? Tal pareciera que siendo tan jóvenes aparentemente sin problemas, se encuentren metidos en este vicio, tal puede ser causante ya que no tienen la responsabilidad de tener hijo, son adolescentes que sus padres los mantienen y los que trabajan no tienen un empleo estresante, la mayoría de los adultos refieren el porqué consumir el cigarrillo y es que 
los adolescentes muchas veces se enfrentan a situaciones todavía más fuertes de superar y por si fuera poco tienen que lidiar día a día con esto motivo por el cual consumen dicha droga, dentro de las principales causas por lo cual consumen esta droga legal Son:

Presión Social: la cual la ejecuta la sociedad sobre un determinado individuo es tan grande que terminan convirtiendo a un estudiante en víctima de estos grandes vicios, sobre todo entre las edades de la pubertad y adolescencia, en donde este tiene la necesidad constante de ser aceptados, está presión es más fuerte que lo que parece, es un sentimiento que reta a los estudiantes, poniendo en juego la duda de su valentía o condicionándolos para ingresar a un determinado grupo de otros compañeros.

Problemas escolares: estos problemas que viven los alumnos, los sumergen en un gran estrés, en una depresión y angustia, que al final terminan por encontrar una salida fácil, los alumnos que fuman encuentran en el cigarro una manera de olvidarse de sus problemas escolares, ¿por qué sucede esto? En realidad, la culpa no es de alguien en particular, pero los casos más comunes y los testimonios de los estudiantes apuntan a los padres, quienes muchas veces en su afán de conseguir mejor calidad de vida, se olvidan de sus hijos dejándolos gran parte del día solos y esto permite que tengan más tiempo de ocio, sumando que no son escuchados y además los regañan constantemente por su mal rendimiento escolar.

Problemas familiares: estos al igual que los escolares crean en el estudiante un sentimiento de depresión, y al buscar salidas podrían terminar siendo adicto al cigarro o a otros vicios, la presencia de de problemas más comunes en la familia son: separación o divorcio de los padres, maltrato físico o psicológico dentro del hogar, discusiones familiares, perdida de un familiar cercano, déficit económico por nombrar algunos.

Problemas personales: también influyen los problemas personales en los estudiantes que fuman mucho, estos problemas están presentes en todas las edades, pero en la adolescencia surge uno en particular que es el enamoramiento, que a su vez trae consigo otras situaciones; el primer amor, obsesiones, embarazos no deseados, amor no correspondido, etc. El adolescente que experimenta por primera vez este tipo de sentimiento es aún más vulnerable a caer en depresión y aferrarse al alcohol y cigarrillo. Abuso físico: cuando existen problemas de bullying o también llamado acoso escolar, aunque estos problemas físicos también podrían suscitarse desde el hogar del estudiante. 
Abuso psicológico: al igual que en el caso, anterior, los insultos, gritos, amenazas y otras situaciones, provocan en el adolescente un temor incontrolable, lo curioso que el cigarro y el alcohol tiene la fama de volver valiente a cualquier individuo.

Curiosidad: algunos estudiantes empiezan a fumar por simple curiosidad, el problema está cuando se acostumbran a ello-

Adicción: aquel estudiante que empezó fumando por cualquier situación que sea y que ahora ya no puede dejarlo, necesita de ayuda, terapia, rehabilitación y sobre todo compromiso para que deje de fumar.

\section{Figura 1}

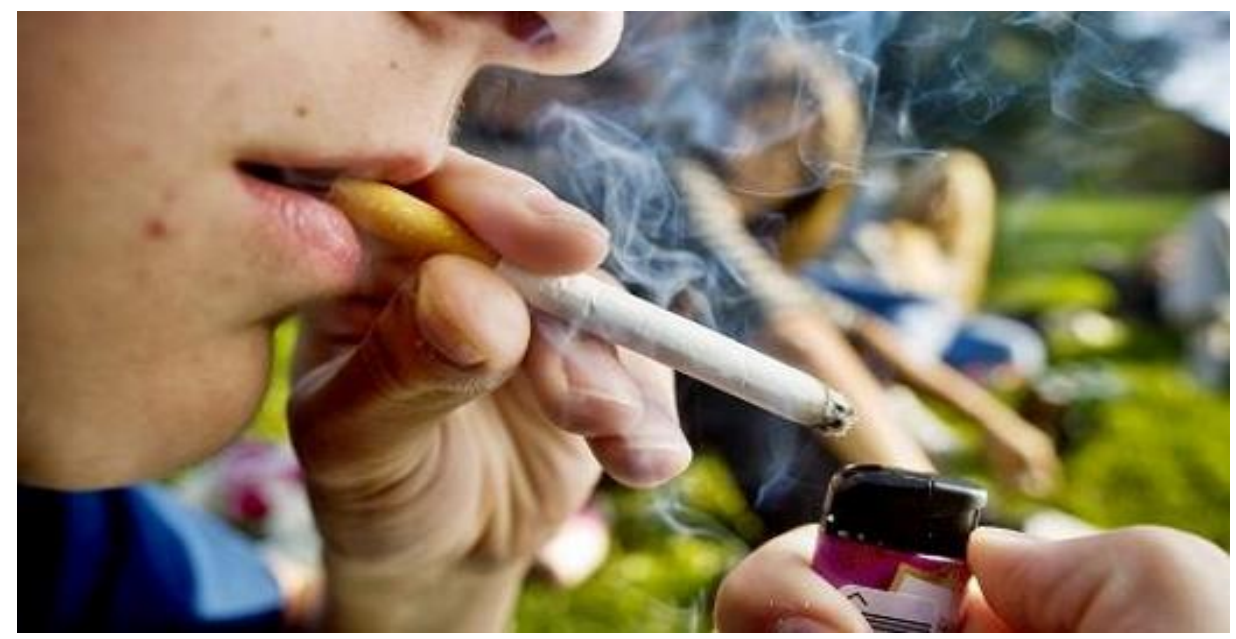

Por su parte, Pilatti et al., (2019), mencionan que, y concordando con lo ya dicho, la percepción de riesgo influye para una orientación hacía el consumo de sustancias, pero agregan otros elementos: la impulsividad y las normas sociales. La primera está relacionada con la personalidad del adolescente, que puede influir en otros aspectos de su vida y no únicamente en el hecho de ingerir cualquier droga, no obstante, la impulsividad la relacionan con la toma de decisiones apresuradas y no planificadas, lo cual explica cómo es que esta puede relacionarse con el consumo de alcohol por parte de los jóvenes, ya que estos beben o fuman por imitación de pares y sin tomarse un momento para analizar las consecuencias de sus actos.

Por otro lado, a las normas sociales las dividen en descriptivas, la percepción del consumo de sustancias en sus iguales; y prescriptivas, la percepción en la aprobación del consumo de sustancias por sus iguales. El resultado de esta investigación nos 
presenta que entre los jóvenes universitarios las normas sociales prescriptivas determinan en gran medida el consumo de alcohol y marihuana, es decir, se asocia la aprobación de otros universitarios con un mayor índice de ingerir estas sustancias (Pilatti et al., 2019).

Con lo antes dicho podemos afirmar que una de las razones principales del consumo de drogas es la inclusión a determinado grupo social, esto es, los adolescentes recurren al uso de estas sustancias para, de alguna manera, lograr sentir la aprobación de sus pares $\mathrm{y}$, por ende, la pertenencia a este colectivo.

\section{Figura 2}

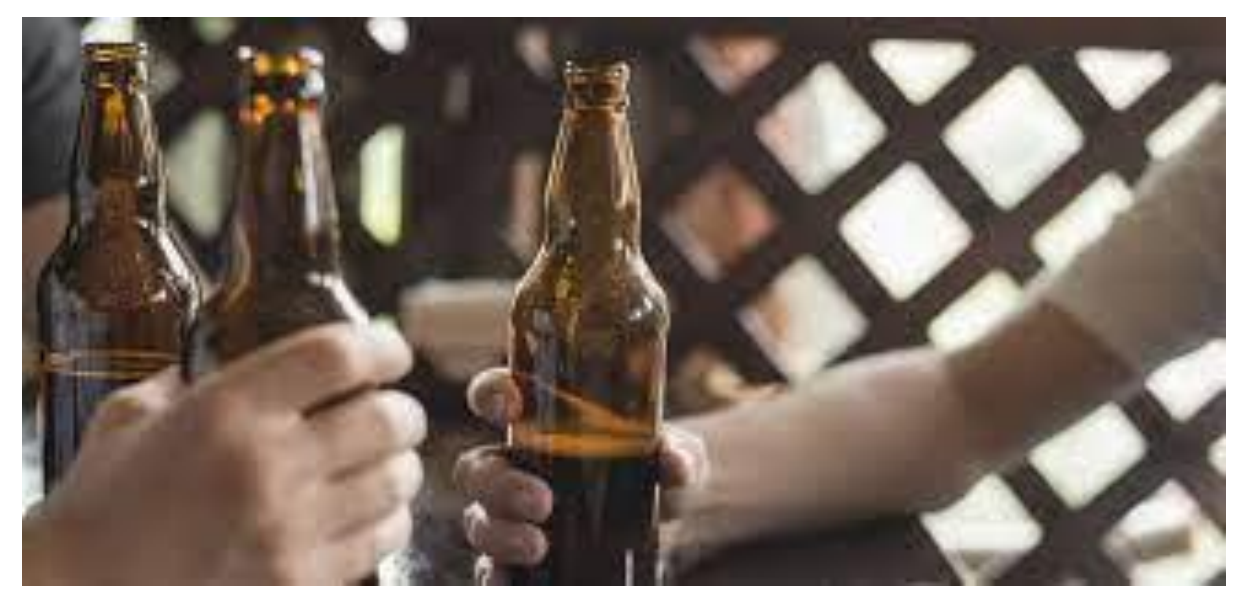

Como ya se observó, hemos realizado un acercamiento a los porcentajes de consumo de drogas en México, las principales sustancias ingeridas por los jóvenes, así como ciertos factores que influyen en la decisión que estos toman para inmiscuirse en este mundo y concordamos que esta problemática afecta de manera directa a los adolescentes y sus vidas. No obstante, aunque son tres las principales sustancias que mencionamos de manera recurrente con anterioridad: alcohol, cigarro y marihuana, debido a la influencia que tienen en los jóvenes, únicamente nos centraremos en las primeras dos, ya que la marihuana aún se encuentra en proceso de legalización, pero no puede considerarse una droga legal aún, por ende, es más difícil su comercialización y distribución. Mientras que, el alcohol y el tabaco, al estar legalizados son sustancias de fácil acceso a las que los adolescentes recurren ya sea para desestresarse, su disfrute, o para convivir, por lo tanto, el objetivo de esta investigación será realizar una aproximación al consumo de drogas legales, así como sus efectos en la escuela preparatoria “José María Morelos y 
Pavón”, la cual se encuentra incorporada en la Universidad Michoacana de San Nicolás de Hidalgo.

\section{MÉTODO}

\section{Participantes}

Esta investigación la llevamos a cabo desde el paradigma cuantitativo, con un enfoque descriptivo transversal. Los participantes que en un inicio consideramos en este trabajo fueron 245 alumnos, inscritos en el último año de preparatoria, los cuales tienen edades comprendidas entre los 17 y 18 años, de estos, 150 fueron de sexo femenino y 95 de sexo masculino; sin embargo, del total mencionado de alumnos, solo participaron 190 estudiantes, 124 mujeres y 66 hombres. Las encuestas se realizaron en las diez secciones de quinto semestre, turno matutino y vespertino, esto es, de los bachilleratos Químico-Biológicas secciones 01,02 y 21; Histórico-Social secciones 01,02 y 21; Económico-Administrativo secciones 01 y 21; e Ingeniería-Arquitectura secciones 01 y 21, de la preparatoria “José María Morelos y Pavón” perteneciente a la UMSNH en Morelia Michoacán.

\section{Instrumento}

Realizamos una encuesta mixta para conocer el alcance del conocimiento que tienen los alumnos acerca de las drogas y sus efectos, donde únicamente tomamos en cuenta seis reactivos:

1. ¿Sabes que el cigarro y las bebidas alcohólicas se consideran drogas legales?

2. ¿Has consumido cigarro y/o alcohol?

3. ¿Cuál de las anteriores es la que consumes con mayor frecuencia?

4. ¿Cuántos cigarros consumes al día?

5.- ¿Cuántas bebidas alcohólicas consumes a la semana?

6. ¿Qué efectos se presentan en tu persona al consumir esta(s) droga(s)?

La manera en la que aplicamos dicha encuesta fue al término de la clase, donde con previa autorización de su profesor, y por medio de Google Meet, nos otorgaron los últimos 15 minutos de su clase, y fue precisamente en la video conferencia que les explicamos en qué consistía la encuesta. De igual manera, les notificamos que su participación no era obligatoria y que esta sería anónima. Recurrimos al programa Microsoft Excel para la elaboración de las gráficas. 


\section{RESULTADOS}

A partir de las encuestas realizadas, encontramos que casi la totalidad de adolescente sabe que existe una clasificación de drogas, con un 97\% confirman que el alcohol y el tabaco se encuentran catalogadas como drogas legales, razón por la cual tienen una mejor recepción, tanto cultural como social, a pesar del daño a la salud que estas pueden generar en los individuos que las consumen (véase Tabla 1).

Tabla 1. Drogas legales.

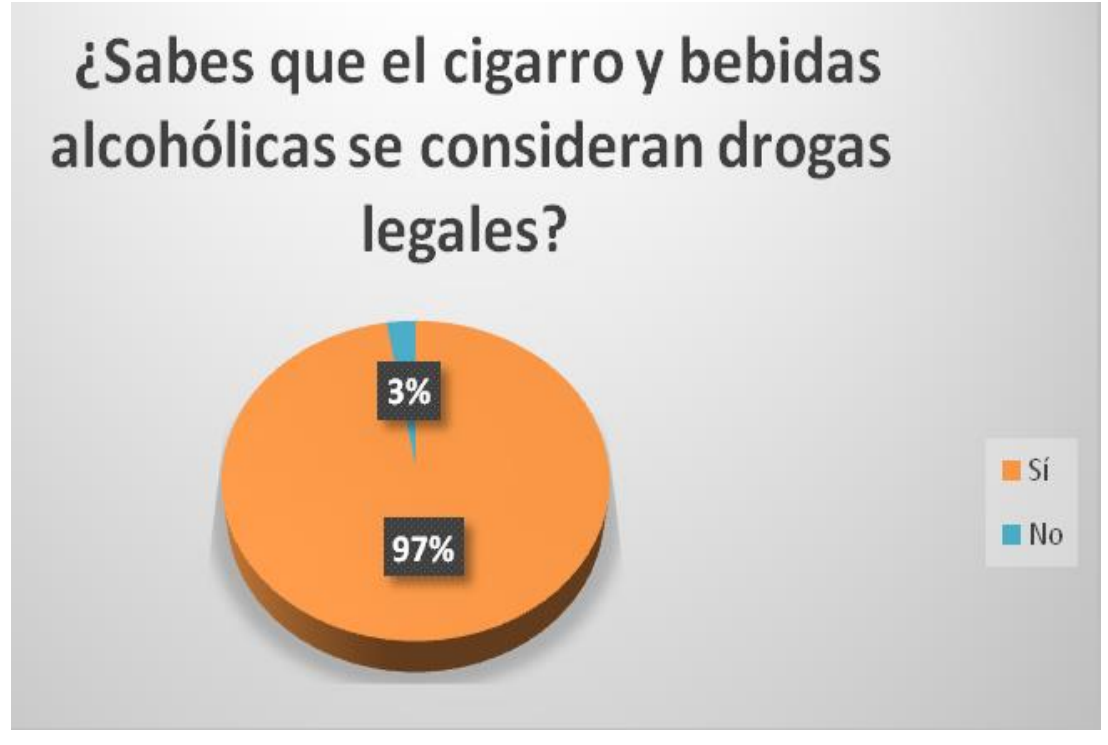

Fuente: Ramírez, Velazco,2020.

Sin embargo, otro resultado de relevancia es que de los 190 alumnos encuestados, el 94\% ha consumido alguna sustancia legal, esto es, la mayoría de los jóvenes que se encuentran estudiando el quinto semestre han probado alguna vez en su vida alcohol o tabaco, lo cual genera un estado de alarma con respecto al consumo de drogas (véase Tabla 2).

Tabla 2. Consumo de alcoho y/o tabaco

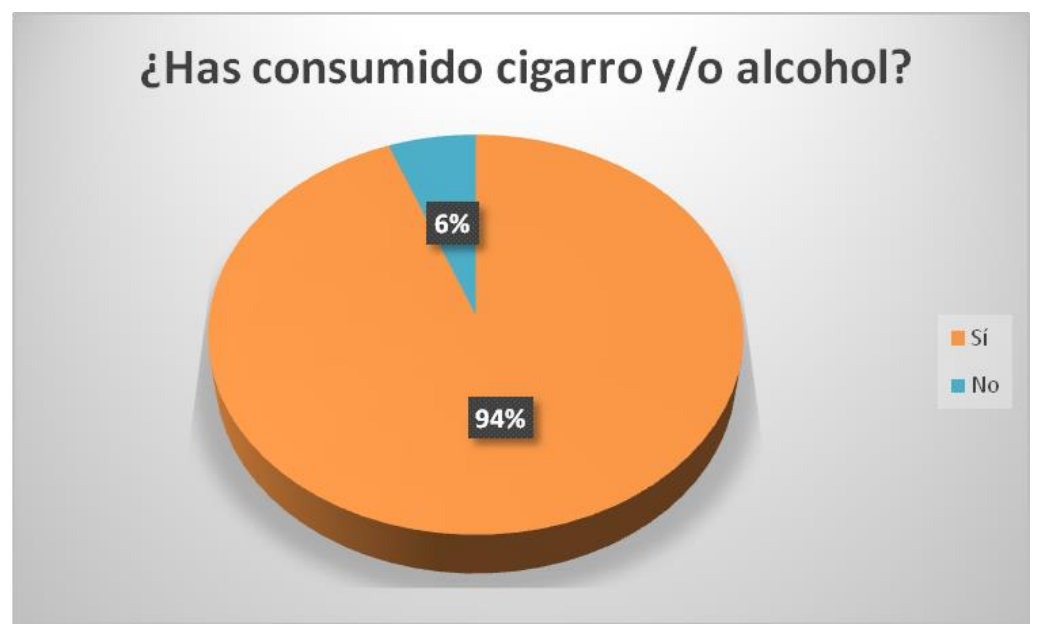


Tabla 3. Droga legal de mayor consumo

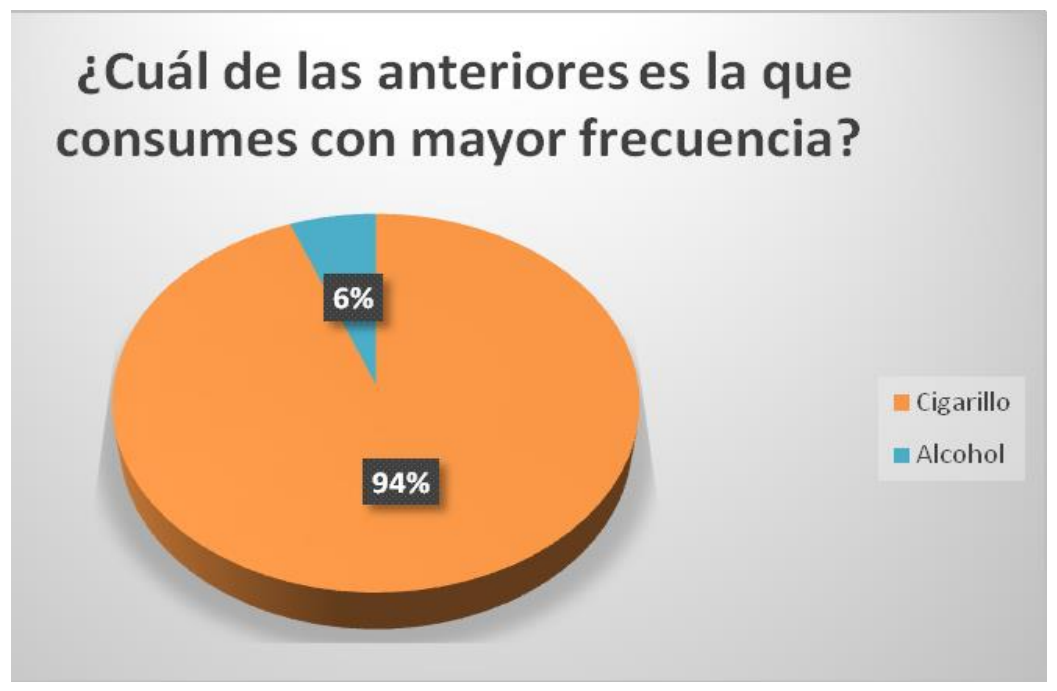

FUENTE: Ramírez, Velazco, (2020).

Con respecto a las sustancias legales más consumidas, podemos observar que el tabaco con un $94 \%$ se ingiere de manera más significante que el alcohol con un $6 \%$ por los jóvenes de esta preparatoria (véase Tabla 3). Y observamos, de igual manera, el número de cigarros que se ingieren en el día, donde el $64 \%$ de los estudiantes fuman por lo menos cuatro cigarros, $23 \%$ fuman dos y el $13 \%$ recurre de uno a dos; mientras que en lo referente al alcohol, encontramos que el 36\% ingiere de 4 a 6 bebidas en la semana, sin embargo, el 64\% de 1 a 2, pero estas se consumen en fiestas o reuniones.

Tabla 4. Cigarros consumidos en un día.

\section{¿Cuántos cigarros consumes al día} cuando estas en la escuela?

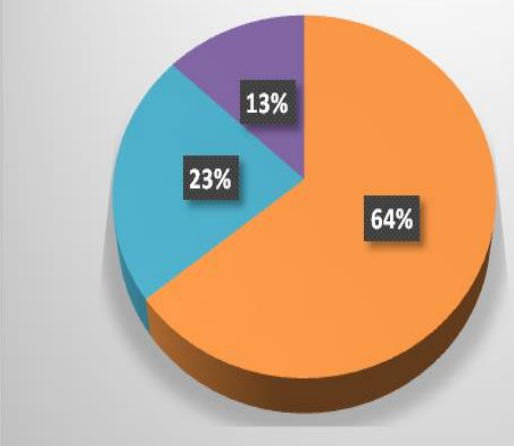

Fuente: Ramírez, Velazco, (2020).
Tabla 5. Bebidas alcohòlicas a la semana.

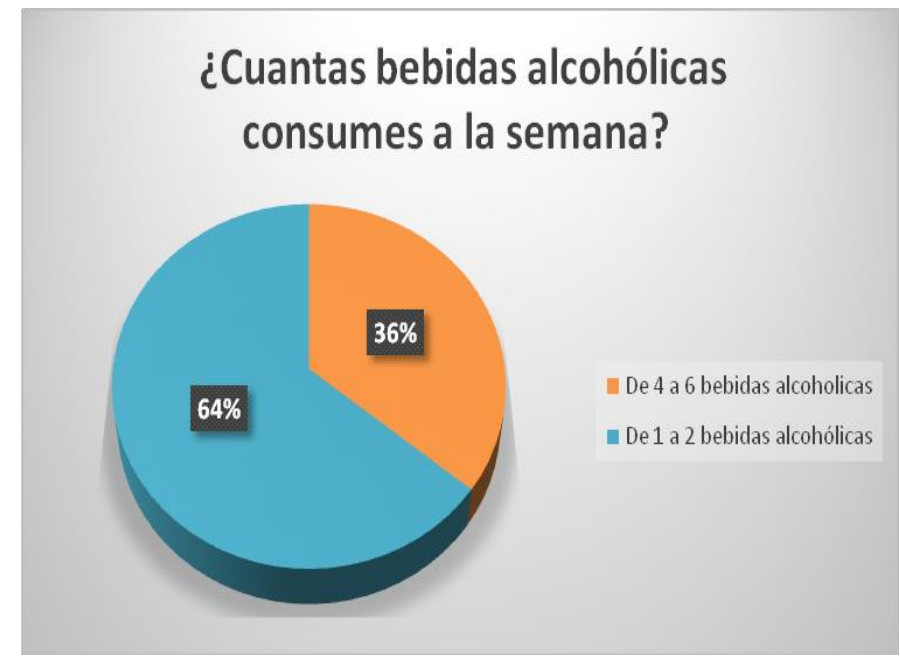

Fuente: Ramírez, Velazco, (2020). 
$\mathrm{Y}$, finalmente, observamos los efectos que se presentan en los adolescentes que consumen este tipo de drogas: el $49 \%$ de los encuestados, casi la mitad, manifiestan que al ingerir, tanto el cigarro como el alcohol, tienen una falta de retención con respecto a lo visto en clases, por ende, se ve afectado su proceso de enseñanza-aprendizaje; mientrás que el $26 \%$ señaló que un efecto que se presenta es la fátiga al hacer ejercicio; y el $25 \%$ restante menciona que la resequedad de garganta es otra consecuencia del consumo de estas sustancias (véase Tabla 6).

Tabla 6. Efectos del consumo de drogas legales

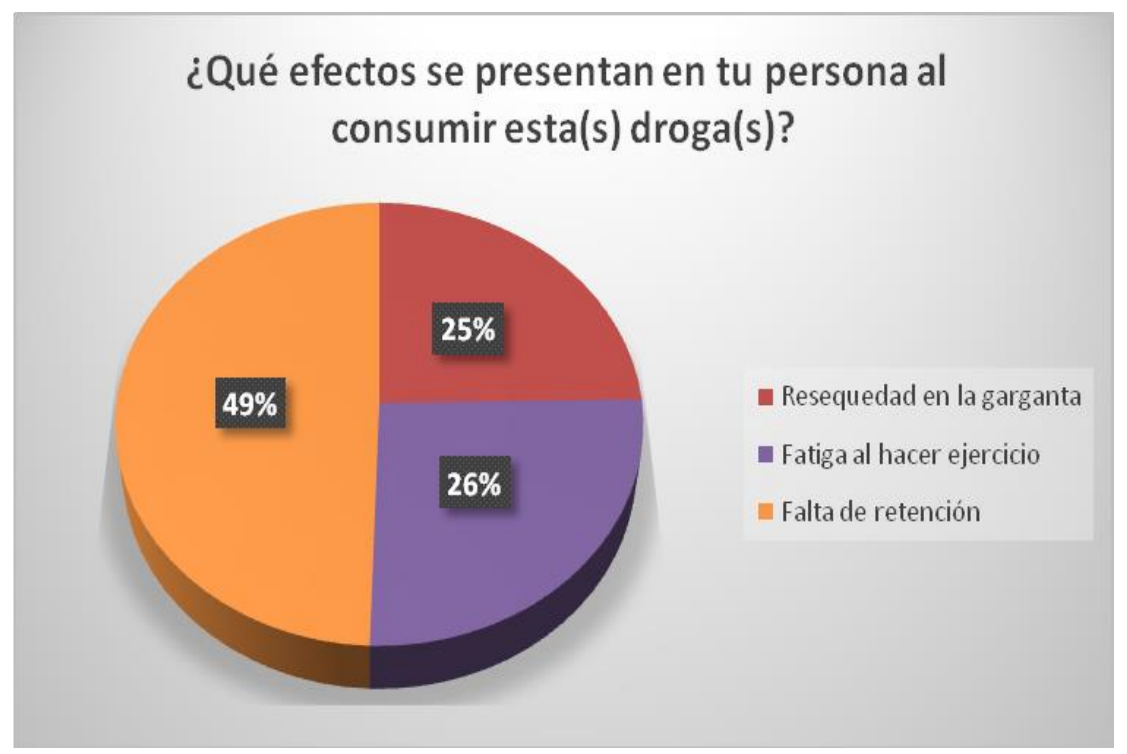

FUENTE: Ramírez, Velazco, (2020).

\section{DISCUSIÓN}

El alcohol y el tabaco, al ser consideradas drogas legales, su venta es màs sencilla, a pesar de que esta sigue siendo limitada, es decir, únicamente aquellos jovenes que sean mayores de edad pueden comprar estas sustancias sin ningún impedimento para ello, pero de acuerdo de al ENCODAT (2016-2017) el consumo de ambas comenza incluso antes de que tengan 15 años, lo que podrìamos describir como ilegal, ya que el uso de estàs no es autorizado sino hasta los 18 .

Si bien hemos dicho que el alcohol es la sustancia màs consumida por adolescentes con un $39.8 \%$ (ENCODAT, reporte de consumo de alcohol, 2017, p. 50), encontramos un cambio significativo en los jòvenes de esta preparatoria, ya que la principal droga legal que se ingiere con mayor frecuencia aquì es el tabaco, superando con un $94 \%$ al alcohol. Lo cual nos sugiere que los adolescentes recurren a los cigarillos por la nicotina, tal y como dicen Barreto et al., basandonse en Goldfrank's Manual of toxicologic 
emergencies, ellos afirman que "el poder adictivo de la nicotina, el alcaloide que contiene, ha servido para asegurar su fuerza y permanencia en el mercado mundial. La nicotina es el principal ingediente psicoactivo que buscan los consumidores de tabaco" (2015, p. 38) debido a los efectos que esta puede producir, ya sea para estimular o relajar.

Este alcaloide, segùn la OMS, produce un efecto de alerta, lo que en algunas personas elevarìa la capacidad de concentraciòn; mientràs que en otras reduce la irritabilidad, asì como la ansiedad (1994, p. 45), esto explica perfectamente el uso recurrente de tabaco que tienen los adolescentes que cursan el quinto semestre de bachillerato, esto es, ellos ingieren los cigarrillos como una manera de disminuir su ansiedad, la cual puede generarse por diversos factores, como lo serìan las clases, exàmenes, trabajos finales o el hecho de ser su ùltimo año antes de ingresar a una universidad, sin embago, y como ya se mencionò antes, puede ser tambien otra forma a la que recurren para concentrarse, que es otro de los efectos que puede provocar la nicotina.

En cuanto al alcohol, si bien es cierto, que su recurrencia es significativamente menor al del tabaco, no significa que sea menos importante. Observamos que de aquellos que ingieren bebidas alcohòlicas, el 36\% llega a tomar de cuatro a seis bebidas en una semana, ante lo cual se encienden las alarmas, debido a que el uso constante de esta sustancia puede derivar en problemàticas màs profundas, entre ellas la dependencia del alcohol, lo cual genera una adicciòn que podrìa, incluso, llegar a ser mortal.

No obstante, el $64 \%$ de los adolescentes recurre a una o dos copas a la semana, y esto sucede, generalmente, en fin de semana social, esto es, en reuniones o fiestas que tienen con sus amigo, colegas, parejas o familiares, lo cual provoca que se ingiera alcohol para sociabilizar con los otros. Con esto regresamos a lo antes dicho por diversos autores: Pillatti et al., 2019; Uribe et al., 2011; asì como Sànchez et al., 2014, los cuales mencionan que un factor importante para el consumo del alcohol es la integraciòn, es decir, sentirnos pertenecintes a determinado grupo imitando su comportamiento, que en este caso serìa ingerir bebidas embriagantes.

Otra de las preguntas realizadas en la encuesta fue: ¿Què efectos se presentan en tu persona al ingerir esta(s) droga(s)? Ante la cual, respondieron que $26 \%$ sentìa fàtiga al hacer ejercicio, lo cual puede provocar a largo plazo otra de las graves problematicas en Mèxico, la obesidad, debido a que al consumir tabaco, principalmente, cuesta màs 
trabajo el respirar mientràs se està realizando alguna actividad fîsica, y como la necesidad de nicotina es bastante importante para los individuos, se prefiere dejar a un lado la condiciòn fìsica.

Otro efecto presentando por los jòvenes es, con un $25 \%$, resequedad de garganta, tomando en cuenta que el cigarro es la principal sustancia ingeridad, lo cual puede provocar esta reacciòn, sin embargo, aunque parece la consecuencia menos grave, esta puede ser muy engañosa, ya que a largo plazo, y si el consumo de cigarros sigue de al menos cuatro al dìa, esta resequedad puede evolucionar y pasar a ser un tipo de cancer relacionado con el consumo de cigarros.

Ahora bien, la respuesta con màs individuos que la secundan es la falta de retenciòn con un $49 \%$. Esto es una problemática a la que no se le debe restar importancia, ya que entendemos que al consumir este tipo de sustancias, incluso siendo drogas legales, afecta de manera significativa su proceso de enseñanza-aprendizaje, lo cual en terminos màs amplios, afecta por completo el futuro de estos jòvenes.

Esta ùltima consecuancia es un factor que se debe tomar en cuanta, ya que al consumir estas sustancias e incluso las drogas ilegales afectan de manera directa el desarrollo escolar de los adolescentes, evitando la retenciòn de informaciòn $\mathrm{y}$, por ende, su vida.

A pesar de que los tres efectos mencionados pueden parecer inofensivos a simple vista, entendemos que a largo plazo pueden traer consecuiencias irremediables en los adolescentes, lo cuales, entre mayor sea su consumo, màs dìficil serà apartarse de ingerir estàs sustancias.

\section{CONCLUSIÓN}

Los datos aquí recopilados nos señalan que las drogas legales son bastante consumidas en la preparatoria “José María Morelos y Pavón” por lo alumnos de quinto semestre, con un $94 \%$ de individuos que han probado el alcohol y/o cigarros, por lo que se propone que se busquen medidas para tratar de detener o, en su defecto, frenar gradualmente el consumo de estas sustancias, las cuales, como ya mencionamos, conforme pase el tiempo las consecuencias podrían ser irreversibles para los adolescentes.

Este es solo un pequeño vistazo a la situación del consumo de drogas legales, que desde el inicio de esta investigación hemos ido tratamos de guiar para saber el alcance que tienen en el país el alcohol y el cigarro, el cual, hasta el momento, se ha mantenido 
estable, e incluso, ha disminuido un poco con respecto al tabaco. Sin embargo, la prevención del consumo de estas sustancias es un trabajo conjunto el cual no se debe dejar de lado bajo ninguna circunstancia, y más al tomar en cuanta las consecuencias que a largo plazo puede traer a los jóvenes.

\section{REFERENCIAS BIBLIOGRAFICAS}

Castells, P. (1999). Relaciones familiares. Barcelona: Ed. STJ.

Castillo, I., Sanz, M., y Pampliega, A. (2004). Funcionamiento familiar y severidad de los problemas asociados a la adicción a drogas en personas que solicitan tratamiento. Adicciones, 16(3). 185-195. Consultado en: http:// adicciones.es/ files/04. \%20Iraurgi.pdf

Centros de Integración Juvenil de México, CIJ, (2007). Violencia Familiar y Adicciones. México D.F.: Autor

Centros de Integración Juvenil de México, CIJ, (2007). Violencia Familiar y Adicciones. México D.F.: Autor.

Cicchetti, D., y Rogosch, F. (2002). A developmental psychopathology perspective on Adolescence. En: Journal of Consulting and Clinical Psychology, 70, 6-20.

Barreto, P., Pérez, M., Roa, M., López, A. \& Rubiano, G. (2015). Consumo de sustancias psicoactivas legales e ilegales, factores de protección y riesgo: estado actual. Revista de Investigación de Salud. Universidad de Boyacá. 2(1). Pp. 3150.

http://revistasdigitales.uniboyaca.edu.co/index.php/rs/article/view/128

Estévez, E., Musitu, G., y Herrero, J. (2005). El rol de la comunicación familiar y del ajuste escolar en la salud mental del adolescente. Salud mental, 28 (4): 81-89. Recuperado de la base de datos Redalyc, (ISSN 0185-3325). http:// redalyc. org/articulo.oa? $\mathrm{id}=58242809$

Galindo, J., Alfaro, I., Osso, L., Mormontoy, W., y Rodríguez, L. (2004). Vulnerabilidad familiar al consumo de drogas en familias adolescentes. Revista de psiquiatría y Salud Mental Hermilio Valdizán, 1, 3-25. Consultado en: www.hhv.gob.pe/ revista/2004/1 \%20VULNERABILIDAD \%20FAMILIAR \%20AL \%20CONSUMO \%20DE \%20DROGAS.pdf

Gómez Cruz, Z., Landeros Ramírez, P., Noa Pérez, M. \& Patricio Martínez, S. (2017). Consumo de alcohol, tabaco y otras drogas en jóvenes universitarios. Revista de 
Salud Pública y Nutrición. 16(4). Pp.1-9. Recuperado de: https://www.medigraphic.com/cgi-bin/new/resumen.cgi?IDARTICULO=76938 Instituto Nacional de Psiquiatría Ramón de la Fuente Muñiz; Instituto Nacional de Salud Pública, Comisión Nacional Contra las Adicciones, Secretaría de Salud. (2017). Encuesta Nacional de Consumo de Drogas, Alcohol y Tabaco (20162017): Reporte de Alcohol. Villatoro-Velázquez, J. A., Reséndiz Escobar, E., Mújica Salazar, A., Bretón-Cirett, M., Cañas-Martínez, V., Soto-Hernández, I., Fregoso-Ito, D., Fleiz-Bautista, C., Medina-Mora, M. E. Gutiérrez-Reyes, J., Franco-Nuñez, A., Romero-Martínez, M. \& Mendoza-Alvarado, L. Ciudad de México: INPRFM.

Instituto Nacional de Psiquiatría Ramón de la Fuente Muñiz; Instituto Nacional de Salud Pública, Comisión Nacional Contra las Adicciones, Secretaría de Salud. (2017). Encuesta Nacional de Consumo de Drogas, Alcohol y Tabaco (2016-2017): Reporte de Drogas. Villatoro-Velázquez, J. A., Reséndiz Escobar, E., Mújica Salazar, A., Bretón-Cirett, M., Cañas-Martínez, V., Soto-Hernández, I., Fregoso-Ito, D., Fleiz-Bautista, C., Medina-Mora, M. E. Gutiérrez-Reyes, J., Franco-Nuñez, A., Romero-Martínez, M. \& Mendoza-Alvarado, L. Ciudad de México: INPRFM.

Instituto Nacional de Psiquiatría Ramón de la Fuente Muñiz; Instituto Nacional de Salud Pública, Comisión Nacional Contra las Adicciones, Secretaría de Salud. (2017). Encuesta Nacional de Consumo de Drogas, Alcohol y Tabaco (20162017): Reporte de Tabaco. Reynales-Shigematsu, L. M., Zavala-Arciniega, L., Paz-Ballesteros, W. C., Gutiérrez-Torres, D. C., García-Buendía, J. C., Rodríguez-Andrade, M. A., Gutiérrez-Reyes, J., Franco-Nuñez, A., RomeroMartínez, M. \&Mendoza-Alvarado, L. Ciudad de México: INPRFM.

León Fuentes JC, Noha Hortal F, Rodríguez Sacristán J. (1990) Reflexiones sobre la situación actual del consumo de drogas por la juventud. Revista Especialidad en Drogas. 15 (1990), pp. 45-51.

Méndez-Ruiz, M. D., de Jesús Alonso-Castillo, M. T., Alonso-Castillo, M. M., UribeAlvarado, J. I. \& Armendáriz-García, N. A. (2015). Relación de percepción de riesgo y consumo de alcohol en adolescentes. SMAD. Revista Electrónica en Salud Mental, Alcohol y Drogas. 11(3). Pp. 161-167. Recuperado de: 
http://pepsic.bvsalud.org/scielo.php?script=sci_arttext\&pid=S1806$\underline{69762015000300007 \& \operatorname{lng}=\mathrm{pt} \& \mathrm{nrm}=\mathrm{iso} \& \mathrm{t} \operatorname{lng}=\mathrm{es}}$

Organización Mundial de la Salud (1994). Glosario de términos de alcohol y drogas. Gobierno de España. Ministerio de Sanidad y Consumo. Recuperado de: https://www.who.int/substance_abuse/terminology/lexicon_alcohol_drugs_spani sh.pdf 106

Organización Mundial de la Salud - OMS (2009). Clasificación Internacional de Enfermedades, 10a Revisión (CIE-10). OMS.

Parada, M. (2009). Consecuencias neuropsicologías del consumo intensivo de alcohol (binge drinking) en jóvenes universitarios (Tesis doctoral no publicada). Universidad de Santiago de Compostela, Santiago de Compostela. Disponible en: http://dspace.usc.es/ bitstream/10347/3388/1/9788498876345_content.pdf6

Pons, J. (1997). El modelado familiar y el papel educativo de los padres en la etiología del consumo de alcohol en los adolescentes. Revista Española de Salud Pública, 72 (3). doi : 10.1590/S1135-57271998000300010

Pilatti, A., Michelini, Y., Rivarola, G., Berberian, M., Carrizo, M. \& Putassi, R. (2019) Consumo de Alcohol y marihuana en universitarios y no universitarios: relación con factores de vulnerabilidad. Quaderns de Psicología. 21(2). Pp. 1-21. Recuperado de: https://www.quadernsdepsicologia.cat/article/view/v21-n2pilatti-michelini-rivarola-etal

Rojas, M. (2005). Consumo de drogas psicoactivas en colectivo de púberes y adolescentes. Pautas y tendencias. Oficina de la Naciones Unidas contra la Droga y el Delito (UNODC) - Centro de Información y Educación para la Prevención del Abuso de Drogas (CEDRO)

Rojas, M., Otiniano, F. y Zereceda, J. (2012). Estudio sobre el consumo intensivo de alcohol (Binge Drinkers) en una población clínica de adolescentes y jóvenes. Lima: CEDRO, documento no publicado

Sánchez Sosa, J. C., Villareal González, M. E., Ávila Guerrero, M. E., Vera Jiménez, A. \& Musitu, G. (2014). Contextos de socialización y consumo de drogas ilegales en adolescentes escolarizados. Psychosocial Intervention. 23(1). Pp. 69-78. Recuperado de: http://scielo.isciii.es/scielo.php?script=sci_arttext\&pid=S1132$\underline{05592014000100008}$ 
Uribe Alvarado, J. I., Verdugo Lucero, J. C. \& Zacarías Salinas, X. (2011) Relación entre percepción de riesgo y consumo de drogas en estudiantes de bachillerato. Psicología y Salud. 21(1). Pp. 47-55. Recuperado de: https://psicologiaysalud.uv.mx/index.php/psicysalud/article/view/586

Vallescar Piñana R, Moreno Aguado V, Sarquella Geli J, Vilar Subiras S, Martín Mateo M,Consumo de tabaco. Estudios comparativo 1992-1986. Gac Sanit 24 (1991), pp. 125-129.

Villatoro Velázquez, J. A., Mendoza Meléndez, M. A., Moreno López, M., Oliva Robles, N., Fregoso Ito, D., Bustos Gamiño, M., Fleiz Bautista, C., Mújica Salazar, R., López Brambila, M. A. \& Medina-Mora Icaza, M. E. (2014). Tendencias del uso de drogas en la Ciudad de México: Encuesta de estudiantes, octubre 2012. Salud Mental. 37(5). Pp. 423-435. Recuperado de: https://www.medigraphic.com/cgi-bin/new/resumen.cgi?IDARTICULO=57060

Villatoro Velázquez, J. A., Medina-Mora Icaza, M. E., del Campo Sánchez, R. M., Fregoso Ito, D. A., Bustos Gamiño, M. N., Reséndiz Escobar, E., Mújica Salazar, R., Bretón Cirett, M., Soto Hernández, I. S. \& Cañas Martínez, V. (2016). El consumo de drogas en estudiantes de México: tendencias y magnitud del problema. Salud Mental. 31 (4). Pp. 193-203. Recuperado de: http://www.medigraphic.com/cgi-bin/new/resumen.cgi?IDARTICULO=67799 